Mediterránea, 5 - Págs. (115 - 128), Año 1981

\title{
CONTRIBUCIÓN SOBRE LAS ODONATOCENOSIS DE LA PROVINCIA DE VALENCIA (ESPAÑA)
}

\author{
Por C. BONET y J.A. GIL-DELGADO ${ }^{1}$
}

Como señala COMPTE (1965), la odonatofauna de la Península Ibérica dista de estar bien conocidad, especialmente en lo referente sobre ciertos aspectos de la biología. Entre estos, la composición de las comunidades de odonatos, cualitativa y cuantitativamente, revisten importancia especial desde el punto de vista ecológico.

Es pretensión nuestra dar a conocer los resultados obtenidos sobre tres cursos de agua de la Provincia de Valencia de las comunidades de odonatos presentes en el transcurso de 1977, así como el tiempo de duración de los imagos.

\section{AREA DE ESTUDIO.}

Los cursos de agua estudiados están localizados en los términos de Estivella, Torrente y en los límites de Alboraya y Almacera, y que discurren a lo largo del Barranco de la Horteta; Barranco del Ca. rraixet; y acequia del Bobalar, ésta última de construcción artificial.

Barranco de

Horteta: En el término de Torrente, en la vertiente sur de la Sierra de Perenchiza y a $19 \mathrm{Kms}$. de la línea costera mediterránea aproximadamente. Se trata de una charca circular en cuyas orillas abundan Juncus spp; Arundo spp; y Typha spp. con entrada continua de agua y sali- 
da por desbordamiento sobre un camino de tierra que cruza el arroyo.

Barranco del

Carraixet: Se escogieron dos estaciones de cien metros de longitud cada una y separadas por un ensanchamiento de la corriente. El agua como en Horteta fluye durante todo el año. A $3 \mathrm{Kms}$. de la línea costera en los márgenes destacan Juncus spp y Arundo spp. A menos de diez metros de altitud el barranco actua de límite entre los términos de Alboraya y Almacera.

Mapa 696. 1:50.000, del S.G.E. 1976 en los UTM YJ2776.

Acequia del

Bobalar: Artificial, construida en la década de los treinta, discurre entre naranjos y almendros en el término de EstiveIla, mapa 668 a escala 1:50.000 del S.G.E. 1975 y en los UTM YJ2799. A $13 \mathrm{Kms}$. de la costa está situado a 160 $\mathrm{m}$. de altítud. Con anchura constante, inferior al metro, el centro está colonizado por Juncus spp y Typha spp. El agua, a pesar de depender de las necesidades de riego de los huertos circundantes, fluye continuamente aunque el nivel de las mismas varía diariamente. La longitud de la acequia es de $700 \mathrm{~m}$.

\section{MATERIAL Y METODO.}

Para conocer las especies de odonatos presentes se capturaron ejemplares para su posterior verificación en el laboratorio. No obstante, ciertas especies de fácil identificación, ej. Anax imperator, no fueron recogidas.

El número de visitas a cada área fue de 47 a la Acequia del Bobalar entre las 10 h. 30' y las 16 h., 31 de 10 h. 30' a 14 h. sobre el Barranco del Carraixet y 5 al Barranco de la Horteta entre las $10 \mathrm{~h}$. y 14 h. 30'. Para completar el catálogo de especies se efectuaron en las dos primeras estaciones mencionadas visitas vespertinas, $17 \mathrm{~h}$. a 21 h. en número de 7 al Barranco del Carraixet y 24 sobre la Acequia del Bobalar.

En cada visita se utilizaba un plano del curso de agua, distinto para cada sesión de trabajo, donde quedaban anotadas las manifestaciones de los individuos pertenecientes a las diferentes especies y señalando sobre el plano el lugar de contacto con cada ejemplar de manera que al final de cada sesión de trabajo era posible cuantificar el número de individuos de las distintas especies, proceso similar al seguido por el método de transectos, censo linear para co- 
munidades de aves (PURROY, 1972). En el Barranco del Carraixet, algunas especies se presentaron en fuertes agregaciones, para ellas los valores que señalemos son aproximativos según las clases 0.10 ; 10-50; 50-100; más de 100 individuos.

\section{RESULTADOS.}

Un total de 22 especies, 12 Zygoptera y 10 Anisoptera, se encontraron en el conjunto de las tres localidades. Su presencia 0 ausencia en las mismas puede apreciarse en la TABLA I. El grado de afinidad entre las tres áreas estudiadas es de 44,4. Tomadas dos a dos Estivella y Torrente presentan mayor afinidad, TABLA II.

La aplicación de la distribución $X^{2}$ (MARGALEF, 1974) al estudio de afinidad entre colectivos, presencia y ausencia, tomadas las localidades dos a dos, señala valores de 1,$8 ; 3,56 ;$ y 1,34 , todos ellos con $p<0,05$ que expresan la carencia de diferencias entre las comunidades estudiadas. $A$ tal fin, similares resultados aparecen tras su aplicación sobre Zygoptera y Anisoptera, TABLA II, tomadas independientemente.

\section{DURACION ANUAL DE LOS IMAGOS.}

La FIGURA 1 presenta el intervalo anual en que las distintas especies se muestran en estado de imago en el Barranco del Carraixet y la Acequia del Bobalar. A nivel del conjunto global de especies la comunidad presente en el Barranco del Carraixet mantiene un periodo de mayor longitud anual con individuos adultos, finales de febrero a noviembre. En Estivella, aunque la desaparición de los imagos ocurre hacia la misma época, los primeros imagos aparecen con un mes de retraso respecto al Barranco del Carraixet. Respecto al Barranco de la Horteta, el exiguo número de visitas efectuadas no permite precisar los intervalos específicos anuales.

Entre las especies bien representadas en las dos localidades, I. elegans, C. tenellum, A. imperator, y C. erithraea, muestran un periodo de vuelo más extenso en el Barranco del Carraixet con aparición anterior y desaparición posterior. Esta norma es también seguida a nivel genérico en Calpteryx, Coenagrion y Simpetrum. La aparición con dos meses de antelación en Estivella de $S$. fusca respecto a los imagos del Barranco del Carraixet de la misma especie constituyeron la excepción. Las restantes especies presentes en las localidades mencionadas en este apartado, TABLA I, fueron puntuales, contactos en una visita.

El período de vuelo más amplio lo mantiene I. elegans sobre el Barranco del Carraixet y coincidente con el indicado para el total de la comunidad. En dicha especie el intervalo obtenido es superior al 


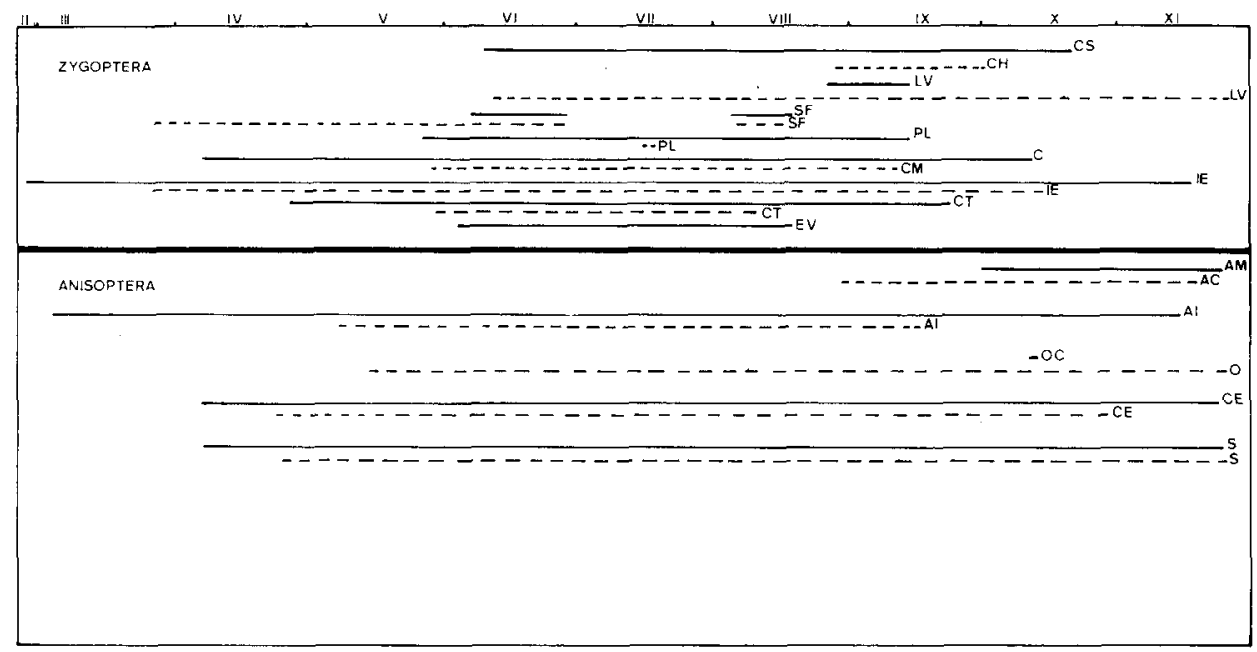

FIGURA 1.-Intervalo anual de vuelo de las diferentes especies sobre el Barranco del Carraixet (línea continua) y la Acequia del Bobalar (línea discontinua).

CS: C. splendens.

$\mathrm{CH}$ : C. haemorrhoidalis.

LV: L. viridis.

SF: S. fusca.

PL: P. latipes.

$\mathrm{C}$ :Coenagrion spp.

$\mathrm{CM}$ : C. mercuriale.

IE: I. elegans.

CT: C. tenellum.

EV: E. viridulum.

AM: A. mixta.

AC: A. cyanea.

Al: A. imperator.

OC: $O$. chrysostigma.

O : Orthetrum spp.

CE: C. erythraea.

$S$ :Sympetrum spp. 
ESPECIE

Calopteryx haemorrhoidalis

Calopteryx splendens

Lestes

viridis

Sympecma fusca

Platycnemis acutipennis

Platycnemis latipes

Coenagrion lindeni

Coenagrion mercuriale

Coenagrion puella

Ischnura elegans

Ceriagrion tenellum

Erythromma viridulum

Gomphus pulchellus

Aeschna mixta

Aeschna cyanea

Anax imperator

Orthetrum chrysostigma

Orthetrum coerulescens

Orthetrum brunneum

Crocothemis erythraea

Sympetrum striolatum

Sympetrum fonscolombei

TOTAL
ALBORAYA ESTIVELLA TORRENTE

$x$

$x$

$x$

$x$

$x$

$x$

$x$

$x$

$x$

$x$

$x$

$x$

$x \quad x$

$x$

$x$

$x$

$x$

14 $x$

$x$

$x$

$\mathbf{x}$

$x$

$x \quad x$

$x \quad x$

$x \quad x$

$x \quad x$

$x$

$x$

$x$

$x$

$x$

$x$

$x$

15

TABLA I.- Distribución de las especies en las tres localidades estudiadas. Alboraya = Barranco del Carraixet. Estivella $=$ Acequia de Bobalar. Torrente $=$ Horteta. La $x$ señala la presencia de la especie, y en el total el número de especies encontradas en cada localidad.

Acequia del

Bobalar

Barranco del

Carraixet

Barranco de la Horteta

\begin{tabular}{c|c|c|}
\hline Acequia del Bobalan & 52,6 & 57,9 \\
& 1,3968 & 0,321 \\
& 0 & 0,6857 \\
\hline & & 45 \\
& Barranco del & 1,5468 \\
Carraixet & 1,0714 \\
\hline
\end{tabular}

TABLA II.-Grado de afinidad entre localidades, valor superior de cada cuadrícula, valores de chi-cuadrado para Zygoptera, cifra media, y Anisoptera, cifra inferior de cada cuadrícula. 
reflejado para otros paises, AGUESSE (1968) para Francia; CONSIGLIO y cols. (1974) en las cercanias de Roma (Italia); y ROBERT (1958) para Suiza. En general y para el conjunto de especies se aprécia esta mayor amplitud en fase imago, con aparición más temprana y desaparición más tardía, bien ambas (ROBERT, 1958; AGUESSE, 1968; CONSIGLIO y. cols., 1974).

\section{ABUNDANCIA ANUAL.}

Los recuentos lineales efectuados en los recorridos por las orillas, permiten cifrar las frecuencias diarias de las distintas especies. En algunos casos, por dificultades de identificación entre especies pertenecientes al mismo género, los resultados vienen expresados a nivel genérico. Durante los transectos lucía el sol y no soplaba el viento, ello con el fin de mantener las condiciones climatológicas constantes. Las frecuencias diarias están basadas únicamente sobre las observaciones matinales.

I. elegans, C. tenellum y Coenagrion spp, aparecen en agregados en el Barranco del Carraixet y en más abundancia los dos primeros citados, FIGURA 2. En este centro, $L$. viridis y $O$. chrysostigma son puntuales al igual que $P$. latipes y $C$. haemorrhoidalis en la Acequia del Bobalar.

El número de individuos de cada especie censados por sesión se muestran en las FIGURAS 2, Zygoptera, y 3, Anisoptera. Las especies puntuales no están representadas.

Zygoptera: E. viridulum, falta en Estivella y su presencia en el Barranco del Carraixet es más o menos constante mientras muestra actividad de vuelo. Otro tanto ocurre con $S$. fusca en ambas localidades, a pesar del intervalo de julio que debe coincidir con la fase de desarrollo larvario indicada en Francia por AGUESSE (1968) para los meses de mayo y junio. Un mes de retraso presente en dicho proceso la especie en las localidades aquí estudiadas.

l. elegans, tiene a principios de mayo una pequeña cima en Estivella, FIGURA 2, menor no obstante que los máximos de agosto a principios de septiembre. Hacia el 20 de agosto puede apreciarse un descenso de individuos que vuelve a recuperarse hasta alcanzar la tercera cima de principios de septiembre. A continuación se inicia el descenso hasta su desaparición a mediados de octubre. Su aparición a finales de marzo ocurre con un mes de retraso en relación con el Barranco del Carraixet, donde una primera y pequeña cima se observa hacia el 20 de abril, con descenso posterior y elevarse a finales de mayo por encima de los 50 individuos, abundancia existen- 


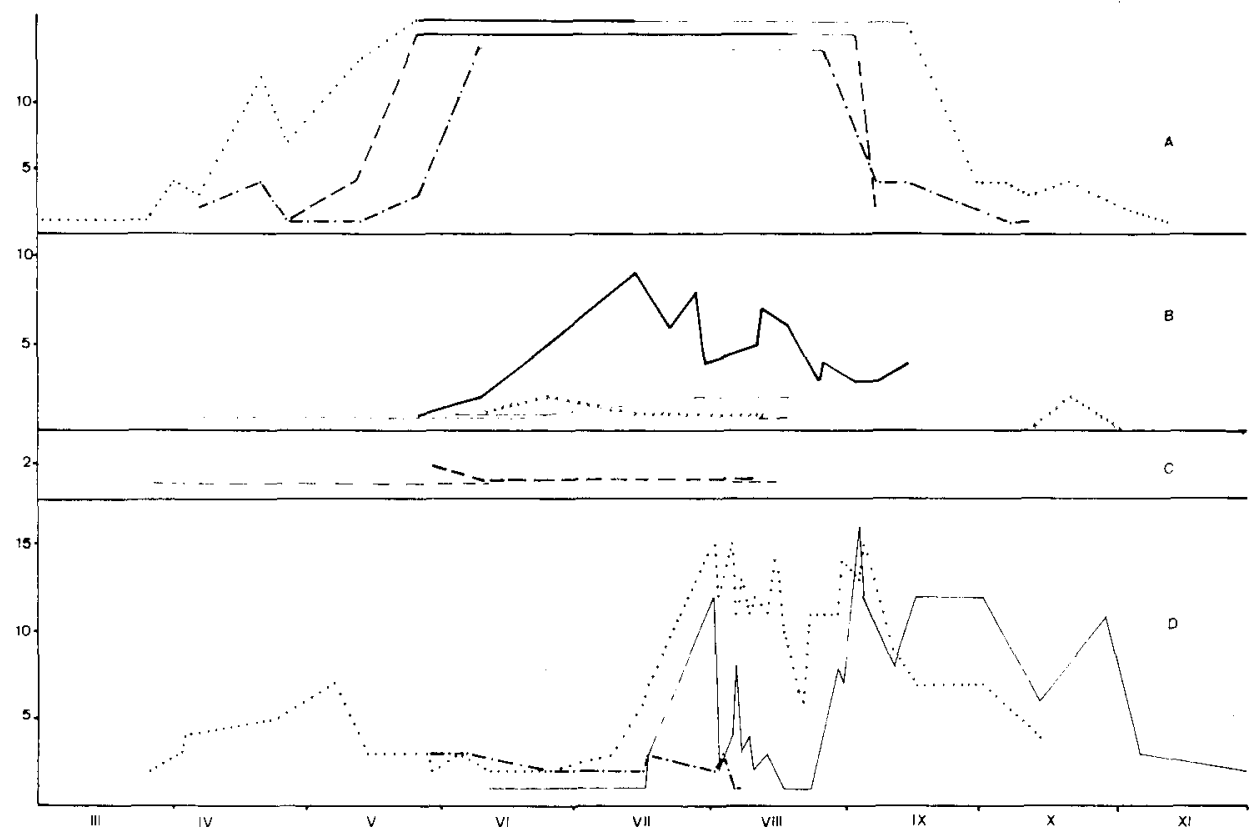

FIGURA 2.-Frecuencia de individuos (Zygoptera) en el Barranco del Carraixet (pisos A y B) y en la Acequia del Bobalar (estratos C y D).

Linea de puntos: l. elegans.

Linea discontinua gruesa: C. tenellum.

Línea discontinua punteada gruesa: Coenagrion spp.

Linea continua gruesa: $P$. latipes.

Línea continua fina: $L$. viridis.

Línea discontinua fina: $E$. viridulum.

Línea discontinua punteada fina: $S$. fusca.

Linea de $\mathrm{x}$ : $C$. splendens.

Los trazos horizontales del estrato A muestran el intervalo en que las especies correspondientes a los trazos mantuvieron más de 25 individuos. 


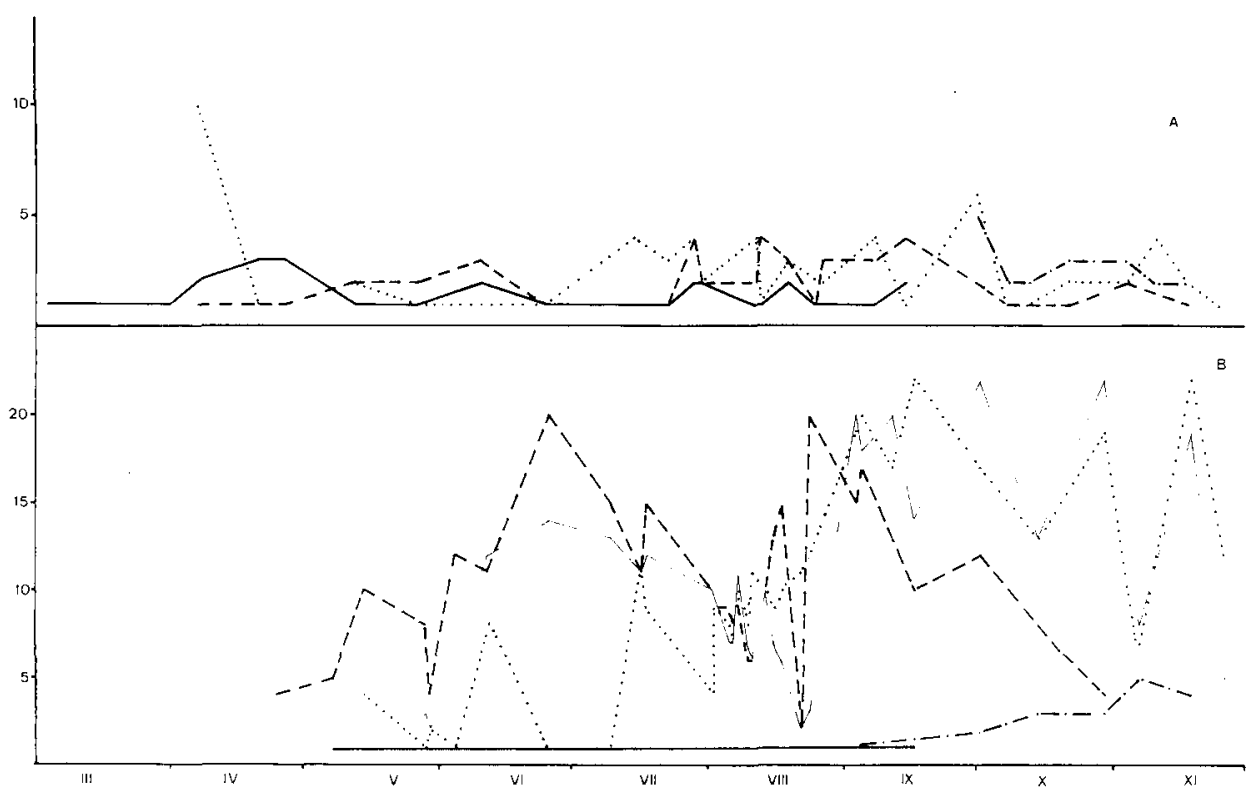

FIGURA 3.-Frecuencia de individuos (Anisoptera) sobre el Barranco de la Horteta (estrato A) y Acequia del Bobalar (estrato B).

Línea continua gruesa: $A$. imperator.

Línea continua fina: Orthetrum spp.

Línea discontinua: $C$. erythraea.

Línea discontinua punteada: Aeschna cyanea.

Línea de puntos: Sympetrum spp. 
te hasta el 14 de julio. No obstante, hasta la primera quincena de septiembre los censos mostraron valores superiores a los quince ejemplares. Desaparecen los últimos individuos un mes después que en la Acequia del Bobalar.

L. viridis, puntual en el área más cercana a la costa, presenta una cima inicial a principios de agosto en EstiveIla, FIGURA 2, a continuación puede apreciarse un descenso de la población hasta la tercera decena de agosto para crecer y a principios de septiembre reflejar los superiores valores. Más abundante durante septiembre y octubre que en los restantes meses que presenta la fase imago. En la Acequia del Bobalar es la especie de desaparición más tardía.

Las especies pertenecientes al género Coenagrion tienen una permanencia de la fase imago más amplia en el Barranco del Carraixet. En Estivella, sólo C. mercuriale y presente únicamente desde fines de mayo a principios de agosto al igual que Ceriagrion tenellum en esta localidad. Las dos especies en el Barranco del Carraixet, TABLA I, desde la primera decena de julio a finales de agosto presentan valores comprendidos entre 10 y 50 individuos. La presencia de imagos duplica en el intervalo anual sobre el Barranco del Carraixet al periodo activo de vuelo en Estivella. Similar modelo presenta C. teneIlum, si bien su desaparición es más temprana que en Coenagrion. C. tenellum es la especie más abundante en el Barranco del Carraixet, una vez que alcanza la cima entre principios de junio y tercera decena de agosto. La frecuencia de individuos y duración de la fase de imago en el resto de las especies existentes en el Barranco del Carraixet puede apreciarse en la FIGURA 2.

Anisoptera: La duración anual de los imagos se muestra en la FIGURA 1, para las localidades en ellas citadas. En el límite de Alboraya y Almacera falta A. cyanea, por otro lado $A$. mixta es puntual en Estivella. Entre las dos localidades puede apreciarse en las FIGURAS 1 y 3 mayor longitud del período de vuelo activo para las especies comunes en el Barranco del Carraixet. Aeschna llega a coexistir con A. imperator en Estivella, donde las especies pertenecientes a los géneros Orthetrum y Sympetrum son más abundantes de septiembre a noviembre. C. erythraea, presenta dos cimas a finales de junio y agosto respectivamente. Sus efectivos hasta el octavo mes del año son superiores a Orthetrum y Sympetrum.

\section{COEXISTENCIA DE ESPECIES.}

Aunque a lo largo del año el número de especies para cada localidad varía entre catorce y quince, TABLA I, el número de ellas que 


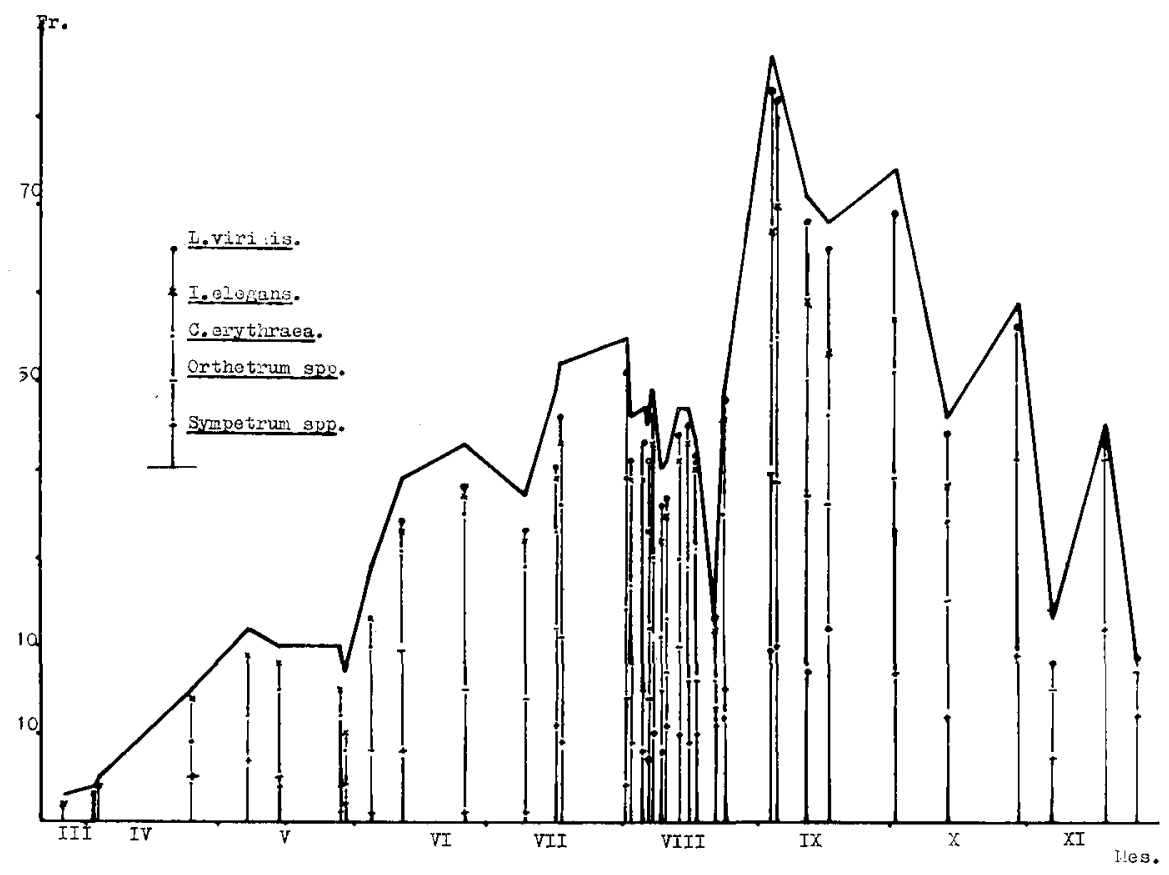

FIGURA 4.-Frecuencias acumuladas de las cinco especies más abundantes en Estivella. La línea gruesa continua expresa el número total de odonatos sobre la Acequia del Bobalar en las distintas visitas. La figura permite apreciar, que es a principios de septiembre cuando aparece el mayor número de individuos sobre el área estudiada. 
$\underset{i}{\frac{1}{x}}+\infty \quad m \propto \infty \quad \frac{0}{0}$

$\sim$ 万ั

$\frac{\pi}{x} N+N+\pi n$

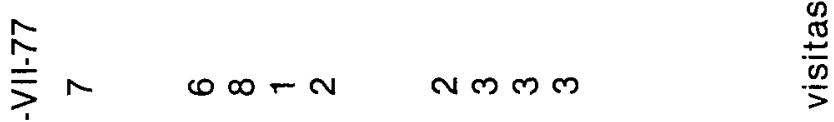

$\frac{O}{\mathrm{O}}$

ธิธี

$\sum_{0}^{N} N+\frac{\widetilde{\infty}}{\sigma}+\quad$ N

c)

$\frac{\Phi}{0}$

$\stackrel{N}{\frac{i}{\operatorname{m}}}$

$\underset{0}{0}$

$\stackrel{0}{0}$

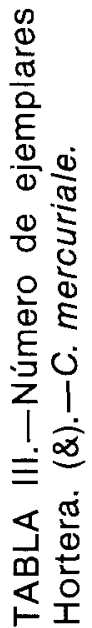


llegan a coexistir es menor, 10 en el Barranco del Carraixet y en Horteta y 9 sobre la Acequia del Bobalar. Los resultados obtenidos en Horteta se muestran en la TABLA III, donde puede apreciarse que el mayor número de individuos que coincide con el máximo de especies coexistentes se da en el mes de septiembre. Este mismo mes mantiene-igualmente mayor abundancia conjunta en Estivella, reflejo de las mayores frecuencias de $L$. viridis, Orthetrum spp y Sympetrum spp, FIGURA 4. En Alboraya, los máximos preceden de junio y julio en función de $C$. tenellum e $\mathrm{l}$. elegans.

El número de especies que llegan a coexistir es similar al señalado para algunas comunidades francesas de Saboya y el Delfinado (DEGRANGE y SEASSAU, 1974) y al máximo obtenido por CONSIGLIO y cols. (1974) en las cercanías de Roma (Italia).

\section{DISCUSION.}

Para las especies no puntuales, el período de vuelo establecido en el conjunto de las tres zonas de estudio, resultan en general superiores a las citadas para otros paises europeos. Así, C. haemorrhoidalis, C. splendens, $L$. viridis, P. latipes, I. elegans, A. mixta, entre otras especies aqui tratadas desaparecen más tardiamente que las citadas de AGUESSE (1968) para Europa Occidental y Norte de Africa. Igualmente, I. elegans y $A$. imperator aparecen con sensible antelación en particular sobre el Barranco del Carraixet.

Respecto a los resultados de COMPTE (1965) para España, C. splendens y $A$. mixta, presentan en el área estudiada desaparición más tardía.

Entre las tres localidades, la más cercana a la costa, marca la ocurrencia de un intervalo anual de vuelo más extenso para el conjunto de la odonatofauna.

En función de la clasificación geonémica establecida por CONCl y NIELSSEN (1956), puede apreciarse que la composición de la odonatofauna está influida principalmente por elementos mediterráneos y euroasiáticos con alguna incrustación etiópica. 


\section{RESUMEN.}

El estudio de tres cursos de agua en tres localidades cercanas a la costa en la Provincia de Valencia (España), muestra la existencia conjunta de 22 especies, 15 en la Acequia del Bobalar (Estivella) y Barranco de Horteta (Torrente) y 14 en el Barranco del Carraixet (Alboraya). No obstante, únicamente 10 llegan a coexistir. La frecuencia mensual para cada especie y para la comunidad en cada localidad es analizada. La composición geonémica de la odonatocenosis está formada por elementos mediterráneos y euroasiáticos.

Las tres comunidades no presentan diferencias, y los períodos de vuelo se mantienen por espacio de tiempo superior que en otros paises europeos.

Conteos lineales fueron utilizados para determinar el número de individuos en cada sesión de trabajo.

\section{SUMMARY.}

The study of three water way in three near sealine localities in the Provincia de Valencia (Spain), shows as a whole 22 species of dragonflies, 15 on the Acequia de Bobalar (Estivella) and Barranco de Horteta (Torrente) and 14 on the Barranco del Carraixet (Alboraya). However, only 10 species coexist.

The monthly frecuency for species and community are analyzed. The communities are composed for Mediterranean and Euroasian members.

There is not differences between the three communities and the flies cycles are more long that other European countries.

Linear census was usabled to determine the individual numbers on each work sesion. 


\section{BIBLIOGRAFIA.}

AGUESSE, P. (1968). Les Odonates de l'Europe Occidentale, du Nord de l'Afrique et des lles Atlantiques. Masson, Paris. 258 pp.

COMPTE, A. (1965). Distribución, ecología y biocenosis de los odonatos ibéricos. Inst. de Biol. Aplicada. 39: 33-64.

CONCI, C. y NIELSSEN, C. (1956). Fauna d'Italia. Odonata. Ed. Calderini, Bologna, $295 \mathrm{pp}$.

CONSIGLIO, C.; ARGANO, A.; y BOITANI, L. (1974). Osservazione ecologiche sugli Odonati adulti di uno stagno dell'Italia centrale. Fragmenta entomologica, 9: 263-281.

DEGRANGE, C. y SEASSAU, M. (1974). Odonates Corduliidae de Savoie et du Dauphiné. Travaux du Laboratoir Hydrobiologique, 64: 289-308.

MARGALEF, R. (1974). Ecología. Ed. Omega. 951 pp.

PURROY, F.J. (1972). Comunidades de aves nidificantes en el bosque pirenaico de Abeto Blanco (Abies alba L.). Bol. de la est. centr. de écol., 1: 41-44.

ROBERT, P.A. (1958). Les libellules (Odonates). Delachaux et Niestlé Neuchâtel. 359 pp. 